\title{
一种新型气相色谱检测器的研究 ${ }^{*}$
}

\author{
于爱民 王䞟 杨文军 金钦议
}

(吉林大学化学系, 长春 130023)

\section{关煡调直流等离子体、㐫子化检测器、气相色漕}

直流等离子体离子化检测器是 60 年代发展起来的. Lovelock 等人首先采用氦气为工 作气体, 研究成功在减压条件下工作的直流等离子体检测器 ${ }^{[1]}$, 引起人们极大的关注. 1987 年 Beres 设计出一种常压下工作的氩直流等离子体离子化检测器, 灵敏度和线性范围比氢火焰离 子化检测器还好 ${ }^{[2]}$. 我国叶芬等人报道的氩直流放电检测器具有很高的灵敏度, 并应用于高 纯气体中杂质组分的测定 ${ }^{[3]}$. 我们在深入研究各种等离子体离子化检测器及其离子化机理的 基础上 ${ }^{[4-61}$, 最近设计组装了一种未见文献报道的新型直流等离子体离子化检测器. 初步实验 证明, 这种检测器对有机化合物和无机气体具有较高的灵敏度, 与已报道的同类检测器相比, 该检测器基流小,噪音低, 等离子体可在较低的电压下稳定地工作. 将该检测器应用于苯乙烯 中乙苯的分析, 测定结果与氢火焰离子化检测器和微波唀导等离子体离子化检测器的结果一 致。

\section{1 实验部 分}

\section{1 检测器结构和工作原理}

检测器的基体采用聚四氟乙烯和石英玻璃材料, 由检测室和放电室构成(见图 1)。由图

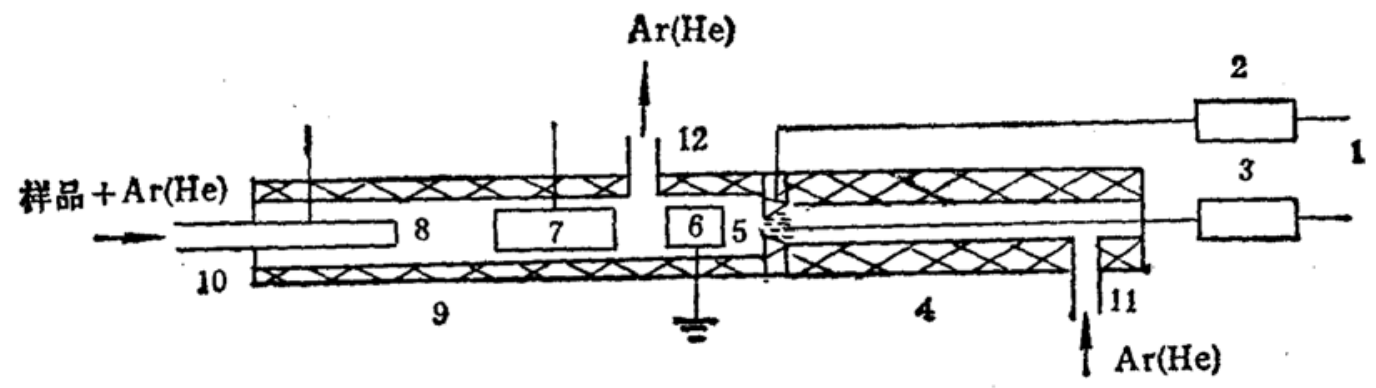

图 1 直流等离子体离子化检测器示意图

1. 接直流电源, 2,3 . 限流电阻, 4. 故电室, 5. 等离子体, 6.铜环, 7. 收集极, 8. 极 化极, 9. 检测公, 10. 接色谱柱, 11. 等离子体工作气体人口, 12. 排气口

1 可见, 放电室中设置二个电极, 阴极为探针式(铂丝), 直径为 $1.5 \mathrm{~mm}$; 阳极为带孔(内径 2

1992-10 26 收稿, 1993-02-10 收修改稿.

*国家自然科学基金资助项目. 
$\mathrm{mm})$ 的铂片。阳极与阴极间距离为 $1.5 \mathrm{~mm}$, 放电室体积为 $0.3 \mathrm{ml}$. 放电室的末端与检测室连 接,检测室中极化极和收集极由不锈钢管组成 (内径分别为 $2 \mathrm{~mm}$ 和 $3 \mathrm{~mm}$ ), 收集极固定在极化 极上方 $5 \mathrm{~mm}$ 处, 而色谱柱的末端直接插入极化极中. 当直流电压通过限流电阻加到阳极和阴 极时, 使流经放电室中的工作气体(He 或 Ar) 在两极之间发生放电, 产生亚稳态原子、分子、激 发态原子和分子等活性组分; 并发射出强烈紫外辐射。这些活性组分具有较高能量,与进人检 测器的被测组分通过电子碰撞电离、Penning 电离或光电离等过程被离子化; 在一定条件下, 通过检测器的被测组分含量与产生离子对的浓度成线性关系, 因而可以通过测量离子流的大 小确定样品中被测组分的含量.

\section{2 检测器的工作条件}

将我们自制的这种新型检测器安装在 SQ-204 型气相色谱仪上,并利用原仪器氢火焰离 子化检测器的极化电压、信号放大和记录系统. 表 1 列出这种新型检测器的工作条件.

表 1 检测器的工作条件

\begin{tabular}{|c|c|}
\hline $\begin{array}{l}\text { 气相色谱仪 } \\
\text { 填充柱 } \\
\text { 工作气体流速 } \\
\text { 载气流速 } \\
\text { 气化温度 } \\
\text { 柱温 } \\
\text { 直流电压 }\end{array}$ & $\begin{array}{l}\text { SQ-204 型, 北京分析仪器厂 } \\
\text { 不锈钢柱 ( } 3 \mathrm{~mm} \times 1 \mathrm{~m}) \text {, 填充 } 3 \% \mathrm{SE} 30,102 \text { 白色担体为固定相 } \\
20-30 \mathrm{ml} / \mathrm{min} \text { ( } \mathrm{He} \text { 或 } \mathrm{Ar} \text { ) } \\
\begin{aligned} 30-50 \mathrm{ml} / \mathrm{min} \\
150^{\circ} \mathrm{C} \\
90^{\circ} \mathrm{C}\end{aligned} \\
750 \mathrm{~V}(\mathrm{He}), 1100 \mathrm{~V} \text { (Ar) }\end{array}$ \\
\hline
\end{tabular}

\section{2 结果与讨论}

\section{1 检测器的性能和特点}

对该检测器的稳定性和样品测定的重复性试验表明,基线漂移不大于 $0.03 \mathrm{mV} / \mathrm{h}$; 对含量 为 $4.4 \times 10^{-9} \mathrm{~g}$ 苯的标样连续平行测定 16 次,所得的相对标准偏差为 $1.3 \%$.

为了进一步考察这种新型直流等离子体离子化检测器的响应特性及其分析性能, 我们对 该检测器的噪声、背叟电流、工作电压、检出限 (对苯)和线性范围等主要性能进行了研究. 并 与国外同类检测器和氢火焰离子化检测器 (FID) 的数据作了对比(见表 2). 由表 2 可见，该 检测器的主要性能指标优于或接近 FID 检测器, 并且一个更重要的特征是这种检测器体积

表 2 检测器主要性能比较

\begin{tabular}{c|c|c|c}
\hline 检 测 器 & 文献 [7] & FID & 本 \\
\hline 背景电流(A) & $6 \times 10^{-9}$ & $2 \times 10^{-11}$ & $1 \times 10^{-11}$ \\
嗓声(A) & $4 \times 10^{-11}$ & $5 \times 10^{-14}$ & $3 \times 10^{-14}$ \\
检出限( $(\mathrm{g}$ 苯) & $4 \times 10^{-11}$ & $5 \times 10^{-11}$ & $6 \times 10^{-12}$ \\
线性范围 & $10^{4}$ & $10^{6}$ & $10^{5}$ \\
\hline
\end{tabular}

表 3 常压宔和氩直流放电工作电压比较

\begin{tabular}{|c|c|c|c|c|c|c|}
\hline 检 & 测 & \multicolumn{2}{|c|}{ 文献 [ 2] } & 文献 [ 3] & 本 & 文 \\
\hline \multicolumn{2}{|r|}{ 工作气体 } & $\mathrm{He}$ & Ar & $\mathrm{Ar}$ & $\mathrm{He}$ & Ar \\
\hline \multicolumn{2}{|r|}{ 工作电任 (V) } & 2200 & 1600 & 1400 & 750 & 1100 \\
\hline
\end{tabular}


小，放电稳定，背景电流小，等离子体工作电压低(见表 3)。这是由于在放电管与检测管之间 设置了排气口和一段铜制金属环 (见图 1), 使等离子体工作气体不经过收集极, 直接从排气口 放空, 减少了工作气体不稳定对信号的影响; 同时金属环与地线相连使等离子体的背景电流 大大降低.

\section{9. 应用}

为了验证本检测器的实用性, 对苯乙烯中乙苯的含量进行了定量测定. 并把本检测器测 得值与 FID 和微波诱导等离子体离子化检测器(MIPID)测得值作了比较, 其测定结果列于表 4.

表 4 苯乙烯中乙苯的测定结果(\%)

\begin{tabular}{c|c|c|c|c}
\hline 样品 & MIPID 法 & F ID 法 & RSD $(\%)$ \\
\hline 1 & 0.19 & 0.18 & 0.19 & 0.8 \\
2 & 0.21 & 0.20 & 0.20 & 0.9 \\
3 & 0.32 & 0.35 & 0.33 & 1.1 \\
4 & 0.26 & 0.29 & 0.28 & 0.7 \\
\hline
\end{tabular}

实验证明, 这种新型检测器的灵敏度取决于等离子体的稳定性和工作气体的种类和纯度. 高纯工作气体有利于灵敏度的进一步提高, 而检测器的稳定性又与放电管的几何尺寸和产生 等离子体所需电压大小有关. 实验发现, 当工作气体为氦气时, 等离子体放电的最佳电压为 $750 \mathrm{~V}$, 当氞气为工作气体时, 等离子体放电的最佳电压为 $1100 \mathrm{~V}$, 由此可见, 在常压直流放电 中, 高电离电位的氦气 $(24.59 \mathrm{eV})$ 比氞气 $(15.76 \mathrm{eV})$ 放电所需的工作电压低得多. 而在常 压微波放电(频率为 $2450 \mathrm{MHz}$ ) 体系中,氩等离子体比氦等离子体更容易获得。可以认为常压 等离子体的形成不仅受工作气体种类及其电离电位大小的影响, 还可能与所用电源的工作电 压和频率有关。虽然在直流放电过程中, 氩气和氦气的解离及其等离子体形成的机理还难以 作确切的描述, 但不影响检测器对被测组分的定量测定.

\section{参文嗝}

[1] Lovelock, J. E.; Anal. Chem., 1961, 33: 163.

[2] Beres, S. A., Halfmnn, D. C., Katz, E. D. et al., Analyst., 1987, 112: 91.

[3] 叶芬、林丛敬、罗春荣等,色谱,1991,9(2): 119.

[4]金钦汉、许宪祝、徐文国,吉林大学自然科学学报, 1990,(2): 71 .

[5] Jin Qin-han, Yang Guang-de, Guo Zhen-ku et al., J. Microchem, 1987, 35: 281.

[6] 于爱民、徐文国、王芬蒂等,高等学校化学学报(英文版), 1991,7(4): 243.

[7] Freeman, R. R., Wentworth, W. E, Anal. Chem., 1971, 43(14): 1987.

[8] Verner, P. J., Chromatogr., 1984, 300:249. 